\title{
ATTI DELLA SOCIETA ITALIANA DI FISICA
}

a eura di G. C. DALLA Noce, Segretario

In seguito a una sovvenzione, che l'Associazione Industriali di Trieste ha recentemente deliberato a vantaggio della nostra società, si rende noto che alla lista dei Premi e Borse di studio per il 1951 posti a concorso con bando del 31 Maggio scorso, pubblicato nel precedente fascicolo del Nuowo Cimento, serie IX, vol. VIII, n. 6, pag. 439.440, deve essere agginnta un'ulteriore

- Borsa di lire 500000 (oinquecentomila) indivisibili, intitolata "Borsa di studio per la Fisica, dell'Associazione Industriali di Trieste ", istituita da detta Associazione.

Le norme del Concorso a questa Borsa sono quelle medesime stabilite per le altre di cui al bando sopra ricordato.

Milano, 15 Giugno 1951.

Il Segretario: G. C. Dalla Noce

Il Presidente: G. PolvanI 